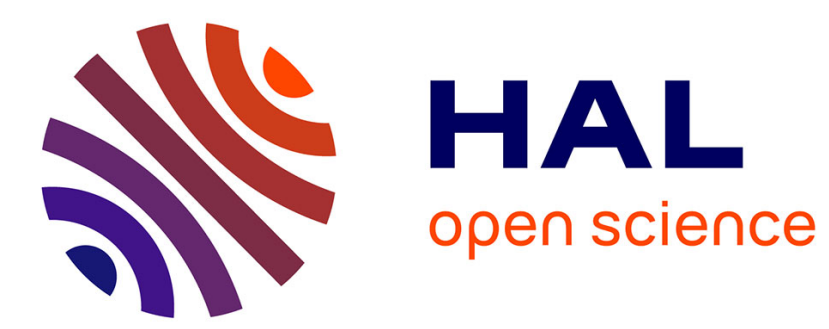

\title{
Trois enfants pour tous en Chine ?
}

Isabelle Attané

\section{To cite this version:}

Isabelle Attané. Trois enfants pour tous en Chine ?. Population et sociétés, 2022, 596, pp.1-4. 10.3917/popsoc.596.0001 . hal-03544698

\section{HAL Id: hal-03544698 \\ https://hal.science/hal-03544698}

Submitted on 26 Jan 2022

HAL is a multi-disciplinary open access archive for the deposit and dissemination of scientific research documents, whether they are published or not. The documents may come from teaching and research institutions in France or abroad, or from public or private research centers.
L'archive ouverte pluridisciplinaire HAL, est destinée au dépôt et à la diffusion de documents scientifiques de niveau recherche, publiés ou non, émanant des établissements d'enseignement et de recherche français ou étrangers, des laboratoires publics ou privés.

\section{(ㄷ)(1) $\Theta$}

Distributed under a Creative Commons Attribution - NoDerivatives| 4.0 International 


\title{
Population \& Sociétés
}

\section{Trois enfants pour tous en Chine?}

\author{
Isabelle Attané*
}

Après avoir longtemps cherché à limiter les naissances, notamment avec la politique de l'enfant unique, le gouvernement chinois les encourage aujourd'hui avec sa nouvelle " politique de trois enfants » instituée en 2021. Isabelle Attané décrit les évolutions démographiques récentes en Chine et examine les changements introduits par cette nouvelle mesure. Permettra-t-elle de ralentir le vieillissement démographique et de pallier le déficit de main d'œuvre ?

Malgré un développement économique spectaculaire au cours des trente dernières années, la Chine a d'importants retards à combler avant de figurer parmi les pays les plus avancés de la planète : en 2020 , elle se plaçait encore au $79^{\mathrm{e}}$ rang mondial pour son PIB par habitant et au $85^{\mathrm{e}}$ pour son indice de développement humain. Mais, alors même que son économie montre des signes d'essoufflement, elle doit en parallèle faire face à des défis démographiques propres aux pays les plus développés : d'une part, une fécondité très basse, passée sous le seuil de remplacement des générations dans la première moitié des années 1990 [1] et tombée à 1,3 enfant par femme en 2020 [2]; d'autre part, une réduction attendue de 70 millions de sa population d'âge actif (20-64 ans) entre 2020 et 2035, dont la part dans la population totale devrait passer de $65 \%$ à $57 \%$; enfin, un vieillissement démographique rapide, la part de personnes âgées de 65 ans ou plus étant susceptible de passer de $12 \%$ à $21 \%$ en quinze ans. La " politique de trois enfants ", annoncée en mai 2021 après la publication des résultats préliminaires du $7^{\mathrm{e}}$ recensement national de la population (2020), vise à atténuer ces tendances. L'objectif est de parvenir, ainsi que l'ambitionne le gouvernement chinois, à un « développement démographique équilibré sur le long terme " permettant de garantir un "développement économique durable » [3]. Le nouvel amendement de la Loi sur la population et la planification des naissances (2002), adopté en août 2021, prévoit diverses mesures visant à lever les obstacles à une remontée de la fécondité, devenus flagrants après l'échec de la "politique de deux enfants » lancée en 2015 (figure 1) : en 2020, la Chine n'a en effet enregistré que de 12 millions de naissances, soit

* Institut national d'études démographiques
Figure 1. Évolution de la fécondité* en Chine selon les sources (2010-2020)

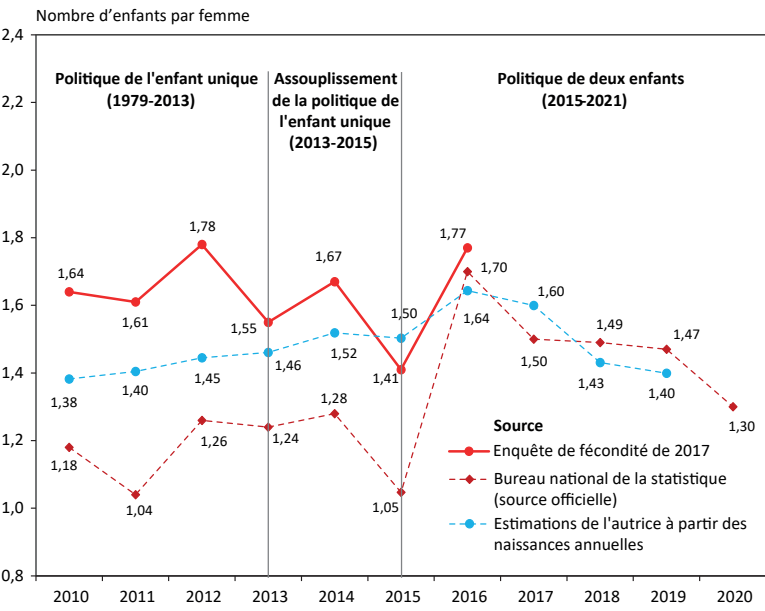

Isabelle Attané, Population \& Sociétés, n 596, INED, janvier 2022.

Sources : voir encadré.

Notes:

1. * indicateur synthétique de fécondité (nombre moyen d'enfants par femme).

2. La politique de l'enfant unique avait été assouplie en 2013 par une disposition autorisant les couples de citadins dont l'un des deux conjoints était lui-même enfant unique à avoir deux enfants, alors que jusque-là, seuls ceux dont les deux conjoints étaient des enfants uniques pouvaient prétendre à cette dérogation [1].

près de 3 millions de moins qu'en 2019 (14,7 millions), et le chiffre le plus bas depuis 1960 - date à laquelle elle comptait deux fois moins d'habitants qu'aujourd'hui. Ces mesures, qui s'apparentent à celles mises en place dans plusieurs pays d'Europe [4], ont pour principal objectif d'alléger les contraintes économiques et matérielles liées à l'arrivée d'un enfant dans 
Tableau. Principaux indicateurs démographiques de la Chine (2010 et 2020)

\begin{tabular}{|c|c|c|c|}
\hline & 2010 & 2020 & Évolution 2010-2020 \\
\hline Population totale (1) (en millions) & 1339,72 & 1411,78 & $+72,06$ millions \\
\hline Taux de natalité (\%) & 11,90 & 8,52 & $-28,4 \%$ \\
\hline Taux de mortalité (\%o) & 7,11 & 7,07 & $-0,56 \%$ \\
\hline Croissance naturelle $(\%)$ & 4,79 & 1,45 & $-69,7 \%$ \\
\hline Enfants (0-14 ans) (en millions et en \% de la population totale) & $222,46(16,2 \%)$ & $253,38(18,0 \%)$ & $+30,92$ millions (soit $+1,3 \%$ par an) \\
\hline $\begin{array}{l}\text { Adultes (15-64 ans) } \\
\text { (en millions et en } \% \text { de la population totale) }\end{array}$ & $998,43(72,9 \%)$ & $967,76(68,5 \%)$ & $-30,67$ millions (soit $-0,3 \%$ par an) \\
\hline $\begin{array}{l}\text { Personnes âgées ( } 65 \text { ans ou plus) } \\
\text { (en millions et en } \% \text { de la population totale) }\end{array}$ & $118,83(8,7 \%)$ & $190,64(13,5 \%)$ & $+71,81$ millions (soit $+4,8 \%$ par an) \\
\hline Nombre moyen de personnes par ménage & 3,1 & 2,6 & $-0,5$ personne \\
\hline $\begin{array}{l}\text { Nombre de femmes en âge d'avoir des enfants (15-49 ans) } \\
\text { (en millions) }\end{array}$ & 379,78 & 338,87 & $-40,91$ millions (soit $-1,1 \%$ par an) \\
\hline $\begin{array}{l}\text { Nombre de femmes aux âges les plus féconds ( } 20-34 \text { ans) } \\
\text { (en millions) }\end{array}$ & 161,20 & 146,03 & $-15,17$ millions (soit $-1,0 \%$ par an) \\
\hline Nombre moyen d'enfants par femme & 1,6 & 1,3 & $-0,3$ point \\
\hline Âge moyen des femmes au premier mariage & 23,6 (en 2006) & 26,3 (en 2016) & $+2,7$ ans \\
\hline Âge moyen des femmes à la naissance du premier enfant & 24,3 (en 2006) & 26,9 (en 2016) & $+2,6$ ans \\
\hline $\begin{array}{l}\text { Rapport de masculinité à la naissance } \\
\text { (garçons pour } 100 \text { filles à la naissance) }\end{array}$ & 117,9 & 111,3 & $-6,6$ points \\
\hline $\begin{array}{l}\text { Rapport de masculinité total } \\
\text { (hommes pour } 100 \text { femmes dans la population totale) }\end{array}$ & 105,2 & 105,1 & $-0,1$ point \\
\hline
\end{tabular}

un couple tout en favorisant l'articulation entre vie familiale et vie professionnelle pour les femmes. L'amendement de 2021 n'énonce cependant que des principes généraux, les modalités de mise en ouvre de ces nouvelles mesures de planification familiale étant, comme cela a été le cas pour les précédentes [5], laissées à l'appréciation des gouvernements des provinces.

\section{Favoriser les naissances}

Le premier amendement de la loi en 2015 avait levé trois freins majeurs à une relance de la fécondité : la limitation à un enfant (ou deux voire trois dans certains cas), l'incitation à retarder le mariage et la procréation (art. 18) et l'obligation de pratiquer la contraception pour les couples en âge d'avoir des enfants (art. 20) [1]. L'amendement de 2021 marque un changement d'approche radical en jetant les bases d'une politique nataliste [6]. Il apporte deux modifications majeures : d'une part, la possibilité d'un troisième enfant offerte à tous les couples, quels que soient leur milieu de résidence (urbain ou rural) ou leur appartenance ethnique ; d'autre part, un changement de langage concernant l'âge au mariage et à la naissance des enfants, qui doit désormais être " approprié ». Bien qu'aucun âge seuil ne soit préconisé à ce stade, il s'agirait donc de contrer la hausse rapide de l'âge moyen des femmes au premier mariage, et donc à la naissance de leur premier enfant (tableau), afin, comprendon, de maximiser le nombre de celles effectivement en couple aux âges les plus féconds. Le caractère non obligatoire de la pratique contraceptive et le libre choix de la méthode employée, introduits par l'amendement de 2015, sont par ailleurs réitérés dans la version de la loi amendée en 2021. La pratique du planning familial " conformément à la loi » reste toutefois une obligation légale (art. 17) et des récompenses - dont la nature n’est pas spécifiée - sont prévues pour les couples qui adhèrent aux nouvelles dispositions (art. 23). Enfin, l'interdiction des échographies et des avortements visant à sélectionner le sexe de l'enfant à naître est maintenue (art. 39). Il s'agit en effet, ainsi que le précise la Décision du Comité central du Parti communiste chinois et du Conseil des Affaires d'État lors de laquelle la "politique de trois enfants " a été approuvée (en date du 26 juin 2021), de tenter de ramener le rapport de masculinité à la naissance à un niveau normal en mettant fin à l'élimination des fotus féminins. Ce rapport, bien qu'en baisse depuis la fin des années 2000 [1], reste en effet très déséquilibré : 111,3 garçons pour 100 filles à la naissance en 2020 (tableau), contre environ 105 attendus dans des circonstances normales.

\section{Faciliter l'articulation entre vie familiale et vie professionnelle}

L'inégale répartition des tâches entre hommes et femmes au sein de la famille et les discriminations des femmes dans le monde du travail comptent parmi les causes de la faible fécondité en Chine [1]. C'est pourquoi l'égalité des sexes, la facilitation de l'articulation entre vie familiale et vie professionnelle et une meilleure protection des femmes sur le marché du travail ont été placées au cœur de l'amendement de 2021. L'article 25 qui, en 2002, prévoyait que « les citoyens retardant leur mariage et la naissance de leur(s) enfant(s) pouvaient bénéficier d'une prolongation des congés de mariage et de maternité » stipule, depuis 2015, que cette prolongation peut bénéficier à toutes les femmes se conformant aux nouvelles dispositions. L'amendement de 2021 réitère la possibilité d'allonger le congé de maternité - sans toutefois préconiser de durée au-delà des 128 jours légaux - et prévoit, ce qui est nouveau, la mise en place d'un congé parental destiné à encourager l'investissement des pères dans le soin de leurs enfants en bas âge [6]. 
Figure 2. Répartition des femmes selon le nombre d'enfants déjà nés et l'âge en Chine (en \%, en 2015)

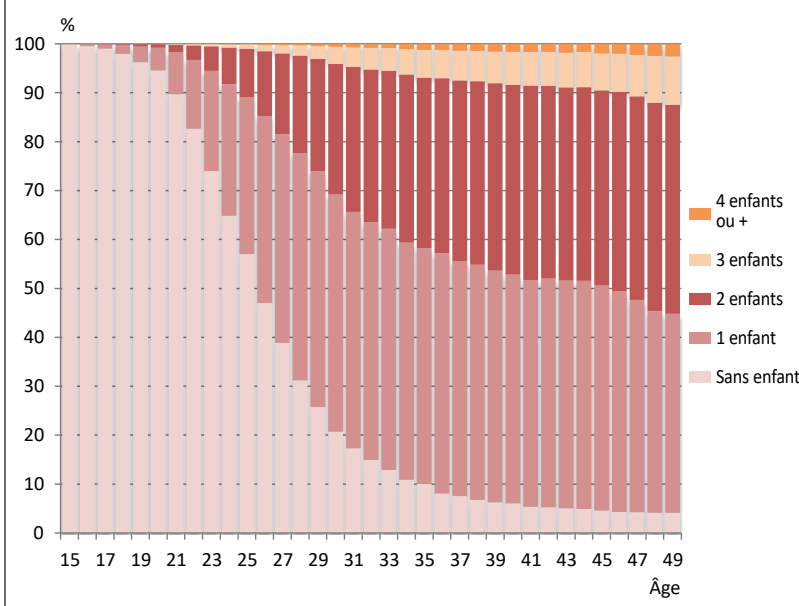

Isabelle Attané, Population \& Sociétés, n 596, INED, janvier 2022. Source : Enquête intercensitaire de 2015

Limiter l'impact d'une naissance sur les possibilités d'emploi, le salaire et la carrière des femmes est une autre préoccupation du gouvernement chinois. Il s'agit en particulier de lutter contre les discriminations des femmes à l'embauche, les inégalités de salaires à poste égal et les licenciements abusifs à la suite d'un congé de maternité, afin notamment de ne pas léser celles qui deviennent mères. Deux aspects de la loi ont été renforcés en ce sens : d'une part, la protection effective des femmes enceintes et des mères sur le marché du travail (art. 26 révisé en 2015 et qui, depuis 2021, stipule que «l'État garantit les droits et intérêts des femmes en matière d'emploi et soutient celles dont la carrière est affectée par la naissance d'un enfant ") et, d'autre part, le développement des services de garde d'enfants en bas âge tant au sein des collectivités locales que des entreprises (art. 28 révisé en 2021). La Décision du 26 juin stipule que les entreprises devront en outre adapter les conditions de travail de leurs salariés (flexibilité des horaires, temps de travail, congés) afin de leur permettre de maintenir un équilibre entre vie familiale et vie professionnelle [3].

\section{Réduire la charge financière pesant sur les familles}

Une autre modification majeure introduite par l'amendement de 2021 vise à alléger la charge financière, souvent rédhibitoire, que représente aujourd'huil'arrivée d'un enfant pour les familles chinoises [1]. Cet allègement sera opéré d'une part en augmentant leurs revenus, grâce au versement de prestations sociales à celles qui ont des enfants « conformément à la Loi » (art. 25) et à la mise en place d'avantages fiscaux (art. 27). Il sera opéré d'autre part en réduisant les dépenses consacrées à la prise en charge de leurs dépendants (enfants et personnes âgées). Enfin, des mesures préférentielles sont également prévues concernant l'attribution d'un logement, l'accès à l'emploi pour les parents et à l'éducation pour les enfants (art. 27). Ces principes généraux
Figure 3. Nombre de naissances selon le rang en Chine, en millions (2010-2020)

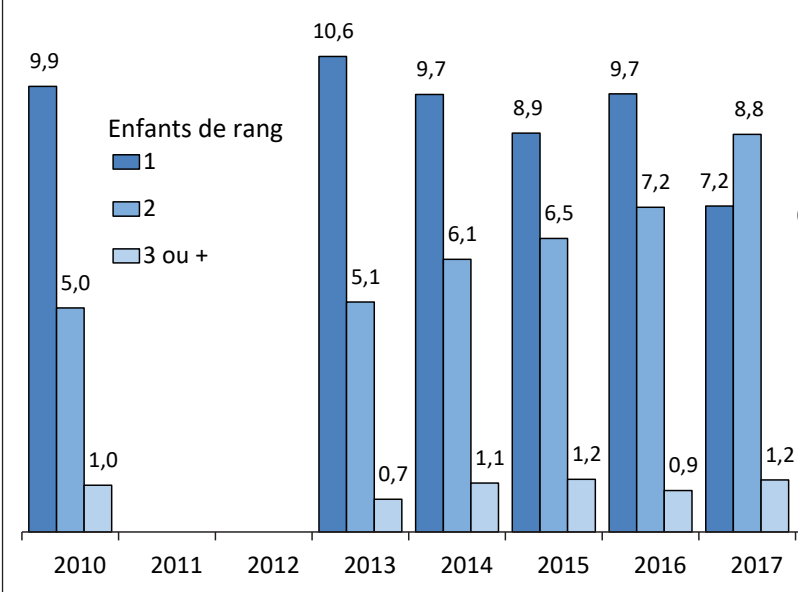

Isabelle Attané, Population \& Sociétés, n 596, INED, janvier 2022.

Sources : Communiqués officiels du Bureau national de la statistique, sources de presse diverses.

seront toutefois soutenus par une reformulation des politiques sociales et fiscales, destinée à favoriser les familles avec des enfants mineurs (notamment ceux âgés de moins de trois ans). Un objectif consiste à réduire les coûts qui incombent aux parents pour la scolarité de leurs enfants (art. 27). Il s'agit notamment, ainsi que le précise la Décision du 26 juin, de normaliser l'accès aux ressources éducatives en le rendant moins dépendant des capacités financières des familles, en particulier en règlementant les enseignements extra-scolaires - source d'importantes dépenses pour les parents qui cherchent à accroître les compétences de leurs enfants dans un système éducatif devenu très élitiste [1]. La Décision du 26 juin 2021 précise également qu'afin d'alléger la charge financière des familles et ainsi leur permettre de centrer davantage leurs dépenses sur leurs enfants, il s'agira de limiter leur implication financière et matérielle dans la prise en charge de leurs parents âgés. Ces mesures devraient inclure un accès prioritaire aux maisons de retraite pour les parents des couples adhérant aux nouvelles dispositions légales, de même qu'une réduction des frais médicaux et des services à la personne [3].

\section{Quels effets peut-on attendre ?}

Cette nouvelle politique, dont les modalités de mise en ouvre au niveau local restent à définir, ne sera cependant pas forcément en mesure de lever tous les obstacles à une remontée de la fécondité, révélant ainsi les limites de l'interventionnisme politique du gouvernement chinois en matière démographique. Si les mesures adoptées au niveau local s'avèrent suffisamment incitatives, elles pourraient certes montrer leur efficacité concernant les arbitrages économiques et familiaux qui, depuis les années 1990 et plus encore que la politique de contrôle des naissances à proprement parler, ont tiré la fécondité chinoise à la baisse (coûts élevés de l'éducation, faible protection des femmes sur le marché du travail, sous-développement des 


\section{Encadré. Sources des données}

Des incertitudes quant au niveau réel de la fécondité en Chine existent depuis les années 1990. Elles sont alimentées par les incohérences relevées entre les différentes sources (recensements, statistiques annuelles des naissances, enquêtes intercensitaires, enquêtes sur la fécondité...) et renforcées par l'opacité des méthodes employées par l'administration statistique pour ajuster I'indice synthétique de fécondité [8]. On relève notamment des écarts importants (autour de 0,5 enfant en moyenne entre 2010 et 2015) entre les valeurs de l'indice synthétique de fécondité présenté comme officiel et celui tiré de l'enquête sur la fécondité de 2017 [9] (figure 1). Les données disponibles sur la natalité et la fécondité restent en outre parcellaires : le Bureau national de la statistique ne publie en effet dans ses annuaires que le taux de natalité, ce qui nécessite de reconstituer les séries annuelles (naissances, indicateur synthétique de fécondité ou rapport de masculinité des naissances) pour la période intercensitaire 20102020 sur la base des informations qu'il communique de manière aléatoire généralement via l'Agence de presse nationale (Agence Chine nouvelle).

infrastructures d'accueil pour enfants en bas âge, perspective de devoir prendre en charge ses parents âgés). En revanche, la nouvelle politique n'aura guère de prise sur les aspirations individuelles des jeunes adultes, qui déterminent fortement leurs comportements de reproduction. L'allongement de la durée des études, notamment pour les femmes, une quête d'épanouissement personnel - qui passe désormais avant le souhait de fonder une famille - ou l'autonomisation vis-à-vis des parents en matière de décisions matrimoniales et familiales, sont autant de facteurs expliquant que les jeunes se marient et font des enfants de plus en plus tard : en effet, si elle reste faible en comparaison d'autres pays de la région, la part de célibataires à 30 ans a plus que quadruplé pour les femmes entre 2000 et 2015 (passant de $2 \%$ à $10 \%$ ) et doublé pour les hommes (passant de $10 \%$ à $20 \%$ ).

L'autorisation d'un troisième enfant échouera aussi à relancer significativement la fécondité à court terme, notamment parce que faire un troisième enfant suppose d'en avoir préalablement fait un premier, puis un deuxième. Or, en 2015, seule une femme d'âge fécond sur quatre avait deux enfants (figures 2 et 3 ); sil'on applique ce pourcentage aux effectifs de celles recensées en 2020 , cela signifie que moins de 85 millions d'entre elles seraient concernées par cette nouvelle possibilité. Bien que l'amendement de 2021 n'apporte aucune précision sur ce point, il est vraisemblable que les mesures incitatives devront bénéficier à toutes les naissances sans distinction de rang, pour cibler notamment les parents d'enfants uniques et les couples sans enfant. En outre, reste à craindre que le gouvernement chinois continue, comme cela a été le cas depuis les années 1970 [5], d'user de la contrainte pour parvenir à ses objectifs en matière démogra- phique. Cet amendement ne comporte en effet aucun signe de relâchement du contrôle susceptible d'être exercé sur les couples. En outre, les avantages accordés à ceux qui se conformeront aux nouvelles dispositions suggèrent que la stigmatisation sociale des autres, de même que les pénalités auxquelles ils pourraient être soumis à l'instar de celles appliquées dans le cadre de la politique de l'enfant unique (amendes, sanctions professionnelles ou administratives, etc.) [5], pourraient perdurer.

\section{Références}

[1] Attané I., 2016, La fin de l'enfant unique en Chine ?, Population \& sociétés, $n^{\circ}$ 535. doi:10.3917/popsoc.535.0001

[2] Communiqué de presse de l'Agence Chine nouvelle, Xinhua wang, 17 mai 2021.

[3] Décision du Comité central du Parti communiste chinois et du Conseil des Affaires d'État, Agence Chine nouvelle, 20 juillet 2021.

[4] Neyer G., 2006, Family policies and fertility in Europe: Fertility policies at the intersection of gender policies, employment policies and care policies, MPIDR, WP 2006-010.

[5] Blayo Y., 1997, Des politiques démographiques en Chine, Paris, Ined- PUF, Travaux et documents.

[6] « Loi sur la population et la planification familiale (amendement du 20 août 2021) », 3 sept. 2021.

[7] Qiao J., Feng H., 2014, Assisted reproductive technology in China: Compliance and non-compliance, Translational Pediatrics, 3(2), 91-97. doi:10.3978/j.issn.2224-4336.2014.01.06

[8] Zhao Z., Zhang X., 2010, La baisse récente de la fécondité en Chine à partir d'une nouvelle reconstitution statistique, Population, 65(3), 513-542. doi:10.3917/popu.1003.0513

[9] He D. et al., 2019, China fertility report, 2006-2016, China population and development studies, 2, 430-439. doi:10.1007/ s42379-019-00022-9

\section{Résumé}

En 2021, la Chine a pour la seconde fois amendé sa Loi sur la population et la planification des naissances pour tenter de relancer la natalité en autorisant les couples à avoir trois enfants. Ce dernier amendement met donc fin à près d'un demi-siècle de contrôle strict des naissances.

La nouvelle politique familiale risque cependant d'échouer à relancer la fécondité à court terme. L'autorisation d'un troisième enfant n'aura en effet que peu d'influence, car faire un troisième enfant suppose d'en avoir préalablement fait un premier, puis un deuxième.

La nouvelle politique ne changera pas les aspirations individuelles de fond des jeunes adultes. L'allongement de la durée des études, notamment pour les femmes, une quête d'épanouissement personnel - qui passe désormais avant le souhait de fonder une famille -, et les inégalités entre les sexes, sont autant de facteurs expliquant que les jeunes se marient de plus en plus tard et font de moins en moins d'enfants.

\section{Mots-clés}

Fécondité, natalité, politique familiale, politique de l'enfant unique, politique de trois enfants, sélection du sexe, vieillissement démographique, Chine.

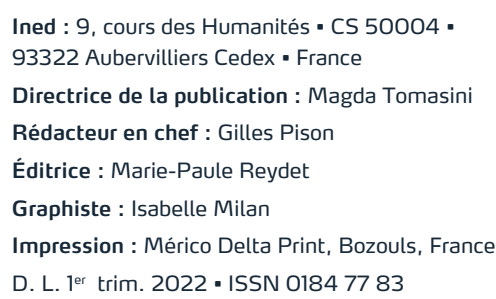

Ined : 9, cours des Humanités - CS 50004 • 93322 Aubervilliers Cedex • France

Directrice de la publication : Magda Tomasini

Rédacteur en chef : Gilles Pison

Éditrice : Marie-Paule Reydet

Graphiste : Isabelle Milan

Impression : Mérico Delta Print, Bozouls, France

D. L. 1er trim. $2022 \cdot$ ISSN 01847783

Numéro 596 - Janvier 2022 - Population \& Sociétés DOI : 10.3917/popsoc.596.0001

Bulletin mensuel d'information de l'Institut national d'études démographiques

Retrouvez Population et Sociétés dès sa parution sur le site internet de l'Ined et abonnez-vous :

wwww.ined.fr/fr/publications/population-et-societes

Contact :

abonnements@ined.fr

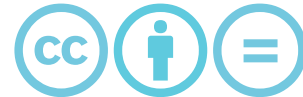

Cet article peut être reproduit sur papier

ou en ligne gratuitement en utilisant notre licence

Creative Commons 